\title{
Education Type
}

National Cancer Institute

\section{Source}

National Cancer Institute. Education Type. NCI Thesaurus. Code C159511.

An indication of the type of schooling an individual receives or received. 